\title{
Demonstration of a Soliton Frequency Comb in a High-Q Silica Microresonator
}

\author{
Xu Yi, Qi-Fan Yang, Ki Youl Yang, Myoung-Gyun Suh and Kerry Vahala \\ Applied Physics, California Institute of Technology, 1200 E California Blvd, Pasadena, CA 91106, USA \\ vahala@caltech.edu
}

\begin{abstract}
Temporal cavity solitons with a detectable repetition rate are generated in a high-Q silica microresonator. A technique for long-term stabilization of the soliton train is demonstrated and used to measure soliton properties for comparison with theory.

(C) 2016 Optical Society of America

OCIS codes: $140.3945,140.4050,190.4380$.
\end{abstract}

The optical frequency comb is revolutionizing a wide range of subjects spanning spectroscopy to time standards [1, 2]. Micro-combs in high-Q microresonators are being pursued as an approach to miniaturization and chip integration of frequency combs [3,4]. A recent advance in this subject has been the demonstration of mode locking and femtosecond pulse generation through the formation of dissipative Kerr solitons in microresonators [5]. Here, we demonstrate soliton mode locking at a detectable repetition rate in a high-Q silica resonator on a silicon chip [6]. Aside from being the first observation of soliton frequency combs in a silica microresonator, the control of soliton properties through a novel locking technique is demonstrated. This technique is used to controllably explore the soliton regime of operation, including measuring the soliton efficiency and existence power, as well as measuring the soliton power and power per comb line versus the soliton pulse width. The measurements are compared with theoretical predictions. The locking technique also enables stable soliton generation indefinitely.

The silica resonators were $3 \mathrm{~mm}$ diameter devices (free-spectral-range of $22 \mathrm{GHz}$ ) with intrinsic Q-factors near 400 million. The mode family used for soliton mode-locking featured anomalous dispersion for optical pumping near 1.55 microns. Moreover, the resonators were optimized so as to produce avoided-crossing-free spectral regions for soliton formation. Solitons form when the pump laser frequency is red detuned relative to an optical mode. The soliton pulse time-domain profile and microwave beatnote were measured using an autocorrelator and electrical spectrum analyzer. The pulse widths were typically in the range of 150-200 fs [6].

For steady-state soliton generation, it was possible to pump the resonator without any frequency locking control of the pump laser. In this mode of operation, solitons were generated for several hours provided that the pump power was high. However, several soliton properties depend upon the pump-resonator relative detuning and it is therefore desirable to stabilize the detuning of the pump relative to the cavity. Because the amount of detuning for soliton operation will typically exceed tens of cavity linewidths, it is not immediately clear how standard locking methods can be applied. Moreover, such techniques may interfere with the mode-locking process itself. The technique demonstrated here uses the theoretical dependence of the average soliton comb power on the pump-resonator detuning [6]. This dependence means that the pump detuning can be servo locked by requiring the comb power to hold a fixed set point. The servo control also has the effect of fixing the soliton pulse width.

Using the locking technique soliton comb properties were measured at a series of detuning values, estimated to range from $12.8 \mathrm{MHz}$ to $29.6 \mathrm{MHz}$, or approximately 13 to 30 cavity linewidths. At each point, the soliton spectrum was also recorded, which enables the calculation of the soliton pulse width. Several soliton properties were studied using this locking technique for the first time. The results of these measurements are compiled in Fig. 1. The soliton power is measured to be inversely proportional to pulse width, while the comb power of the central comb line is independent of pulsewidth (see Fig. 1(a)). These results agrees well with theoretical predictions using measured resonator parameters. Also, Fig. 1(b) shows the soliton spectrum measured at the detuning limits presented in Fig. 1(a). The soliton minimum existence pump power and efficiency were also investigated. To measure the minimum existence power, the pump detuning was held constant while the pump power was reduced until the soliton pulse train disappeared. Both the power level and the soliton spectrum were recorded near the point of disappearance. The pump detuning was then reset to a new value, and the measurement was repeated. Figure 1(c) gives the minimum power measured this way and the soliton nonlinear efficiency plotted versus the pulse width (as calculated from the soliton spectrum). At shorter pulse widths, the measurements deviate from Lugiato-Lefever equation as Raman self-frequency shifts become signicant. It is shown 

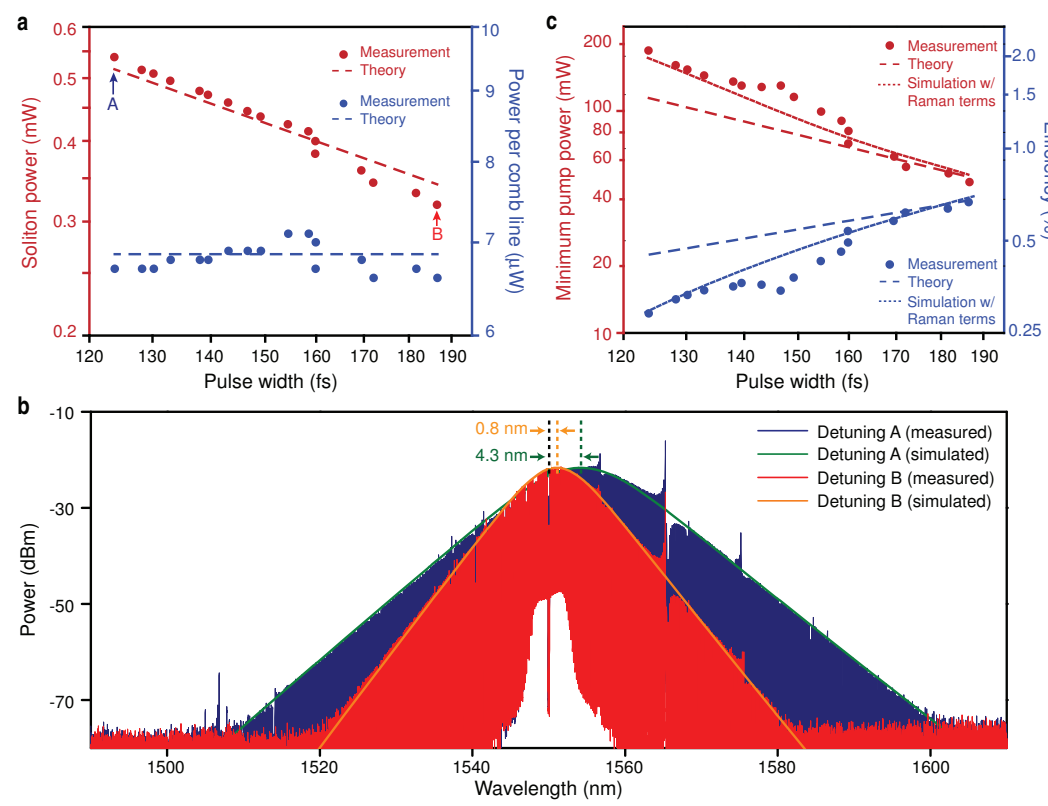
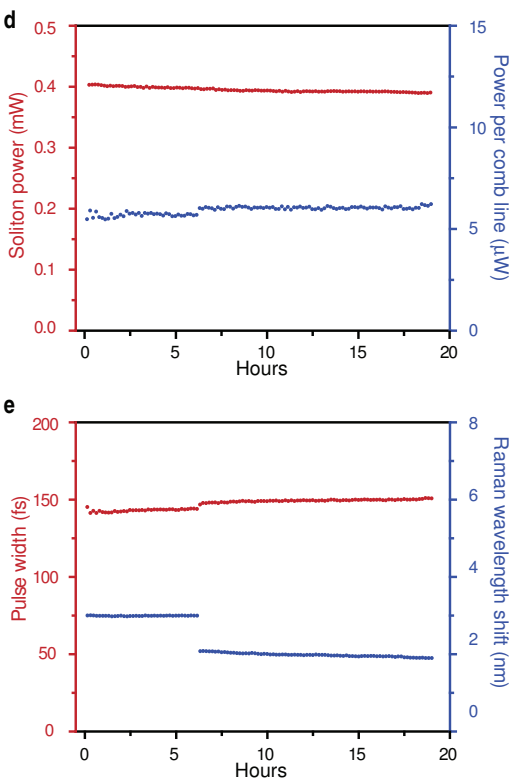

Fig. 1. Demonstration of control of soliton properties. (a) Measured soliton comb output power (red points) and measured power per central comb line (blue) are plotted versus measured soliton pulse width (red points) with comparison to theory (dashed lines). (b) The observed soliton spectra at the limits of the measurement in panel (a) are shown [see arrows A and B in (a)]. Solid orange and green curves are simulations using the Lugiato Lefever equation, including Raman terms. The location of the pump line for both spectra is indicated by the dashed black line and has been suppressed by filtering. (c) The measured minimum pump power for soliton existence (red points) and measured efficiency (blue points) are plotted versus the measured soliton pulse width with comparison to theory (dashed line) and the simulation using the Lugiato-Lefever equation including Raman terms (small dashed lines). (d,e) Continuous soliton measurement over $19 \mathrm{~h}$ using the locking technique. The soliton power, central comb line power, pulse width and Raman wavelength shift are plotted versus time in hours.

that the simulation augmented with the self-frequency-shift Raman interaction agrees well with the measurement (see Fig. 1(c)).

An important benefit of the feedback control technique presented here is that it provides a convenient way to stabilize the mode-locked system indefinitely. For example, the system was operated continuously for over $24 \mathrm{~h}$. For $19 \mathrm{~h}$ of this period, comb properties were recorded and are presented in Fig. 1 (d) and (e).

In summary, we demonstrated soliton mode-locking at a detectable repetition rate in a high-Q silica microresonator. A servo locking technique is shown to maintain the soliton pulse train indefinitely and enabled measurement of soliton properties for comparison with theory.

\section{References}

1. S. Cundiff and J. Ye, Rev. Mod. Phys 75, 325 (2003).

2. S. A. Diddams, et al, Science 306, 1318 (2004).

3. Del'Haye et al, Nature 450, 1214 (2007).

4. T. J. Kippenberg, R. Holzwarth, S. A. Diddams, Science 332, 555 (2011).

5. T. Herr and et al, Nat. Photon, 8, 145 (2014).

6. X. Yi, Q. Yang, K. Yang, M. Suh, K. Vahala, Optica, 2, 1078 (2015). 